\title{
A New Index of Tumor Molecular Heterogeneity for Describing Tumor Occurrence and Development at the Molecular Level
}

\section{Zhao Pengfei}

Dalian Medical University

\section{Luo Hong}

Dalian Medical University

Hui Liu ( $\sim$ liuhui60@sina.com )

Dalian Medical University

\section{Research Article}

Keywords: tumor markers, tumor differentiation, molecular marker, tumor heterogeneity

Posted Date: January 19th, 2022

DOI: https://doi.org/10.21203/rs.3.rs-1264780/v1

License: (c) This work is licensed under a Creative Commons Attribution 4.0 International License.

Read Full License 


\section{Abstract}

Background: To describe an index of tumor occurrence and development at the molecular level based on a series of tumor markers (AFP, CEA, CA19-9, CA72-4, NSE, FER, and CRP)

Methods: The tumor markers were analyzed in patients with poorly differentiated cancer, welldifferentiated cancer, benign lesions, and no benign lesions (control group). Cancer molecular index (CMI) was calculated by multiplying the highest elevation in the tumor marker levels with the number of positive markers.

Results: An increase in the number of abnormal biomarkers was observed with a decrease in the degree of differentiation. A significant relationship was found between $\mathrm{CMI}$ and degree of differentiation $(\mathrm{R}=$ $0.647, P<0.001)$. Based on these findings, benign lesions, well-differentiated tumors, and poorly differentiated tumors may represent a continuous process at the molecular level. In a patient who is positive for certain biomarkers and does not have abnormal imaging findings, a CMI of $<1.35$ can be considered to indicate benign lesions and a $\mathrm{CMI}$ of $>1.35$ can be considered to indicate early-stage tumors or "tumors" that occur at the molecular level.

Conclusion: The CMI system could have potential benefits for describing tumor occurrence and development at the molecular level.

\section{Background}

Cancer is a multi-stage process that involves DNA damage response, proliferation, aging, angiogenesis, and other major cellular pathway changes and defects, and it is usually accompanied by prolonged chromosomal instability. These molecular pathways can be used to distinguish malignant tissues from healthy tissues and make diagnoses and prognostic judgments [1-3]. However, by the time the body exhibits signs of cancer, it has typically already reached the advanced stage. Therefore, in order to diagnose cancers early on, it is important to understand the molecular changes that occur before and simultaneously with the occurrence and development of tumors [4-6]. Although the clinical manifestations of tumors in different parts of the body are different, the molecular mechanisms underlying their occurrence are similar and could be worth exploring from the viewpoint of improving the possibility of early diagnosis.

Tumor molecular markers represent specific cellular mechanisms or tissue functions. In clinical practice, it is often found that a patient is positive for a certain tumor marker but does not have any abnormal imaging findings. This is not surprising, as tumor molecular markers are likely to appear before morphological changes become evident. This initial stage is referred to as the molecular change stage of tumorigenesis (Figure 1). In this stage, tumor molecular markers are likely to be the only indicators of the presence of a tumor. Therefore, studying the molecular changes and characteristics of tumors during their occurrence and development could help determine the nature, characteristics, and prognosis of the tumor before the morphological (pathological) changes occur and diagnose the tumor early on. Currently, 
research on tumor molecular markers has shown considerable progress, and the known tumor molecular markers such as alpha fetoprotein (AFP) [7], carcinoembryonic antigen (CEA) [8], carbohydrate antigen 19-9 (CA19-9) [9], carbohydrate antigen 72-4 (CA72-4) [10], neuron specific enolase (NSE) [11], and ferritin (FER) [12]. These tumor markers are applicable to most tumors and can be considered to represent the molecular level changes that occur in the pre-tumor stage [13].

During the growth process of a tumor, after multiple rounds of division and proliferation, daughter cells may start to show differences in their molecular biology or genetic characteristics. This phenomenon is referred to as tumor heterogeneity. Heterogenous tumors contain several different genotypes or subtypes of cells that cause differences in tumor growth rate, invasion ability, drug sensitivity, prognosis, and other aspects [14-16]. Tumor heterogeneity is one of the characteristics of malignant tumors [17-19]. Studies have shown that the commonly used tumor molecular markers can indicate heterogeneity [13]. In fact, combined detection of multiple tumor markers can improve the sensitivity and specificity of diagnosis of heterogenous tumors [20-22].

Based on the concept of tumor molecular heterogeneity, the present study constructs a tumor differentiation index for recognizing and describing tumor occurrence and development at the molecular level, and understanding the relationship between changes in tumor molecular markers and histopathological changes that occur during the development of tumors.

\section{Material And Methods}

\subsection{Participants}

Cancer group: A total of 110 patients ( 56 with well-differentiated cancer and 54 with poorly differentiated cancer) in stage I or higher stage according to the TNM classification system were recruited as the tumor group. Of the 56 patients with well-differentiated tumors, 17 had lung cancer; 34 , gastrointestinal tract and esophageal cancer; 3 , liver cancer; and 2, other cancers. Among the 54 patients with poorly differentiated cancer, 10 had lung cancer; 32, gastrointestinal tract and esophageal cancer; 4, liver cancer; and 8 , other cancers. The inclusion criteria were (1) evidence of cancer based on surgical and pathologic findings and (2) collection of samples before surgery and in the absence of any drug treatment. The exclusion criteria were (1) incomplete medical records and (2) presence of comorbidities such as hypertension, coronary heart disease, diabetes mellitus, and nephrosis.

Benign lesion group: A total of 52 patients with cysts or polyps ( 11 with cysts, 10 with polyps, and 31 with cysts and polyps) were recruited as the benign lesion group. The cysts or polyps were evidenced by ultrasound examination or imaging, and cancers were excluded based on biopsy examination. The exclusion criteria were the same as those for the cancer group.

Control group: The control group comprised 31 individuals who were selected based on routine physical examinations. The inclusion criteria were absence of benign lesions, such as cysts and polyps, confirmed 
by ultrasound examination or imaging, and absence of comorbidities such as cancer, hypertension, coronary heart disease, and diabetes mellitus.

The age and sex of the four groups are shown in Table 1.

The samples were collected from the First Hospital of Dandong, China. All the subjects were unrelated ethnic northern Han Chinese individuals. The experiments on the subjects were conducted in accordance with the Declaration of Helsinki. The blood taken was as part of usual care for the patients rather then research purposes. Institutional Ethics Committee of Dalian Medical University approved this study.

\subsection{Measurement of tumor markers}

The following tumor markers routinely measured in at our clinical laboratory were selected: CEA (0-4.5 $\mathrm{ng} / \mathrm{ml})$, AFP (0-7.0 ng/ml), CA19-9 (0-30 IU/ml), CA72-4 (0-7.0 lU/ml), NSE (0-16.5 IU/ml), FER (Men: 27-375ng/ml; Women: $12-135 \mathrm{ng} / \mathrm{ml})$, and C-reactive protein, CRP $(0-8 \mathrm{mg} / \mathrm{L})$. These tumor markers were measured in the serum of all study participants by a chemiluminescent microparticle immunoassay with Mindray i2000 device (Mindray, Ltd., China) using standard commercial reagent kits.

\subsection{Cancer molecular index}

The ratio of the sample test value and the cut-off value, which will be henceforth referred to as the S/Co value, was determined for each tumor marker. The S/CO values of different tumor markers were comparable. For each patient, the highest $\mathrm{S} / \mathrm{Co}$ value (Max[S/Co]) from the S/Co values of all the tested tumor markers was multiplied with the number of molecular markers that were detected $(\mathrm{N})$ to determine the cancer molecular index (CMI).

$\mathrm{CMI}=\operatorname{Max}(\mathrm{S} / \mathrm{Co}) \times \mathrm{N}$

Theoretically, only one $\mathrm{CMI}$ can be calculated for each cancer patient at one point of time.

\subsection{Statistical analysis}

Data showed a non-parametric distribution and were reported as the median and interquartile range. The significance of the differences was determined by Kruskal-Wallis $\mathrm{H}$ tests for abnormally distributed continuous data. Chi-squared test was used to test the significance of the differences for count data. Spearman's correlation analysis was used to evaluate the correlation. Receiver operating characteristic (ROC) curves were plotted using the CMls to in order to estimate diagnostic efficacy and optimum cutoff value. All statistical analyses used SPSS software (SPSS, Chicago, IL, USA), and a value of $P<0.05$ (bilateral), was considered as statistically significant.

\section{Results}

\subsection{Tumor molecular marker data}


The raw data for the seven tested tumor markers in the four groups are shown in Table 2. There were significant differences between the groups with regard to six of the seven markers $(P<0.05)$.

The positivity rates of the analyzed tumor markers in the four groups are shown in Table 3. The positivity rate of all seven tumor markers did not exceed $5 \%$ in the control group. There were significant differences among the groups with regard to the positivity rate of all seven tumor markers $(P<0.05)$.

The percentage of individuals in each group who were positive for $0,1,2,3,4,5,6$, and all 7 biomarkers is shown in Table 4. The number of biomarkers with abnormal levels was found to increase as the degree of differentiation decreased.

\section{$3.2 \mathrm{CMI}$ values and cut-offs for diagnosis}

The CMI values in the four groups are shown in Table 5. CMI was significantly associated with the degree of differentiation $(R=0.647, P<0.05)$.

Receiver operating characteristic curves were plotted to estimate the diagnostic efficacy and optimum cutoff values for CMI. The area under the curve values were $0.932,0.874$, and 0.751 for the poorly differentiated, well-differentiated, and benign lesion groups, respectively (Fig. 2). The optimum cutoff values were 1.0 for benign lesions, 1.35 for well-differentiated tumors, and 9.00 for poorly differentiated tumors, as shown in Figure 3.

\section{Discussion}

The present study presents a new index for the diagnosis of tumors in various states of differentiation, including benign lesions. Our findings also shed light on the relationship between the degree of malignancy (represented by the degree of differentiation) of tumors and changes in tumor-related markers, which are important for the early detection of tumors. The results showed that the seven tumor markers selected in this study had a good correlation with the degree of differentiation of the tumors and benign lesions (Table 3). This means that these tumor markers were appropriate as indicators of tumor differentiation status.

The development of normal cells into tumor cells is characterized by active cell proliferation and changes in cell morphology, function, metabolism, and behavior. These changes are usually reflected in the degree of differentiation of the tumor: the lower the degree of differentiation, the lower is the degree of similarity between tumor cells and normal cells and the higher is the degree of malignancy. At the molecular level, the development of normal cells into tumor cells is accompanied by abnormal molecular-level regulation, and this leads to increased expression of tumor molecular markers and an increase in the number of abnormal molecular markers. Accordingly, in our study, the elevated level of tumor markers and the number of positive tumor markers in the poorly differentiated group were significantly higher than those in the well-differentiated group. Due to the different mechanisms involved in tumor molecular marker 
generation, this result also indicates that the poorly differentiated group is characterized by higher tumor heterogeneity and a higher degree of malignancy at the molecular level.

Based on our analyses of the tumor molecular markers, we calculated CMI by multiplying the highest elevation in tumor markers with the number of abnormal molecular markers. The results show that $\mathrm{CMI}$ is highly correlated with the tumor status, as it was significantly different between the control group, benign lesion group, well-differentiated group, and poorly differentiated group. The CMI values also indicate a continuous molecular-level process underlying the transition of benign lesions to well-differentiated and then poorly differentiated tumors. The above process is the result of abnormal cell regulation and abnormal differentiation, so CMI may act as an early and direct indicator of the occurrence, development, and outcome of tumors. Based on our findings, a CMI value of $<1$ can be considered to indicate normal tissue; a CMI value of 1-1.35, benign lesions; a CMI value of 1.35-9.00, well-differentiated tumors; and a $\mathrm{CMl}$ value of $>9.00$, poorly differentiated tumors.

The CMI construct established in this study can also be used as an independent dimension to describe the characteristics of tumors at the molecular level, and combined with morphological and imaging analysis, CMI can be used to comprehensively assess tumors. Therefore, CMI might be an important parameter, especially in cases where there is no pathological or imaging evidence of a tumor. In a patient

who is positive for only one biomarker that is detected at low levels, if $\mathrm{CMI}$ is $<1.35$, benign lesions can be considered. If the $\mathrm{CMI}$ is $>1.35$, early-stage tumors or "tumors" that occur at the molecular level can be considered and the patient should be closely followed up.

One of the major limitations of this study is that $10 \%$ of the poorly differentiated tumors had a CMI of zero. This is probably because the tumor markers measured do not fully cover the heterogeneity of the tumor. The discovery of more tumor markers may improve the efficiency of the CMI system and help in the comprehensive description and analysis of tumors at the molecular level. Despite the above limitations, we believe that the $\mathrm{CMI}$ construct will prove highly useful for the timely detection of cancer and should be investigated in larger samples of patients to confirm its efficacy.

\section{Conclusion}

In conclusion, the CMI system could have potential benefits for describing tumor occurrence and development at the molecular level. In a patient who is positive for certain biomarkers and does not have abnormal imaging findings, a CMI of $<1.35$ can be considered to indicate benign lesions and a $\mathrm{CMI}$ of $>1.35$ can be considered to indicate early-stage tumors or "tumors" that occur at the molecular level. The $\mathrm{CMI}$ can also be used as an independent dimension to describe the characteristics of tumors at the molecular level.

\section{Abbreviations}


AFP: alpha fetoprotein; CA19-9: carbohydrate antigen 19-9; CA72-4: carbohydrate antigen 72-4; CMI: cancer molecular index; CRP: C-reactive protein; FER: ferritin; NSE: neuron specific enolase

\section{Declarations}

\section{Ethics approval and consent to participate}

Not applicable

\section{Consent to publish}

Not applicable

\section{Availability of data and materials}

The data used to support the findings of this study are available from the corresponding author upon request.

\section{Competing interests}

None declared

\section{Funding}

This work was supported by the Special Grant for Scientific and Technological Innovation of Dalian (grant number No. 2018J12SN084) and Special Grant for Translational Medicine, Dalian Medical University (grant number No. 2015008).

\section{Authors' Contributions}

Z.P. performed the research and acquisition of data; L.H revising the article; L.H. conceived the analysis and wrote the final version of the manuscript. All authors have read and approved the manuscript.

\section{Acknowledgments}

We would like to thank the native English speaking scientists of Elixigen Company (Huntington Beach, California) for editing my manuscript.

\section{Reference}

1. Stanta G, Bonin S. Overview on Clinical Relevance of Intra-Tumor Heterogeneity. Front Med (Lausanne). 2018;5:85.

2. Gentric G, Mieulet V, Mechta-Grigoriou F. Heterogeneity in Cancer Metabolism: New Concepts in an Old Field. Antioxid Redox Signal. 2017;26(9):462-485. 
3. Testa U, Castelli G, Pelosi E. Lung Cancers: Molecular Characterization, Clonal Heterogeneity and Evolution, and Cancer Stem Cells. Cancers (Basel). 2018;10(8):248.

4. Chan DKH, Buczacki SJA. Tumour heterogeneity and evolutionary dynamics in colorectal cancer. Oncogenesis. 2021;10(7):53.

5. Guang Y, Yuzhong L, Hui L. A grading system from health to death using routine experimental indicators based on the pre-chronic disease status theory. BMC Geriatr. 2020;20(1):250.

6. Wenping S, Ying L, Yuzhong L, Hui L. Variations in laboratory parameters in prechronic disease to determine disease occurrence at the molecular level. Biomark Med. 2019;13(14):1227-1234.

7. Zhang J, Chen G, Zhang P, Zhang J, Li X, Gan D, Cao X, Han M, Du H, Ye Y. The threshold of alphafetoprotein (AFP) for the diagnosis of hepatocellular carcinoma: A systematic review and metaanalysis. PLoS One. 2020;15(2):e0228857.

8. Hao C, Zhang G, Zhang L. Serum CEA levels in 49 different types of cancer and noncancer diseases. Prog Mol Biol Transl Sci. 2019;162:213-227.

9. Luo G, Jin K, Deng S, Cheng H, Fan Z, Gong Y, Qian Y, Huang Q, Ni Q, Liu C, Yu X. Roles of CA19-9 in pancreatic cancer: Biomarker, predictor and promoter. Biochim Biophys Acta Rev Cancer. 2021;1875(2):188409.

10. Li M, Xue F, Yang J, Pan X. Correlation between tumor marker CA72-4 and prognosis of patients with gastric cancer: A protocol for systematic review and meta-analysis. Medicine (Baltimore). 2020;99(52):e23723.

11. Yan HJ, Tan Y, Gu W. Neuron specific enolase and prognosis of non-small cell lung cancer: a systematic review and meta-analysis. J BUON. 2014;19(1):153-6.

12. Alkhateeb AA, Connor JR. The significance of ferritin in cancer: anti-oxidation, inflammation and tumorigenesis. Biochim Biophys Acta. 2013;1836(2):245-54.

13. Hui L, Rixv L, Xiuying Z. A system for tumor heterogeneity evaluation and diagnosis based on tumor markers measured routinely in the laboratory. Clin Biochem. 2015;48:1241-5.

14. Lawson DA, Kessenbrock K, Davis RT, Pervolarakis N, Werb Z. Tumour heterogeneity and metastasis at single-cell resolution. Nat Cell Biol. 2018;20(12):1349-1360.

15. Kroeger PT Jr, Drapkin R. Pathogenesis and heterogeneity of ovarian cancer. Curr Opin Obstet Gynecol. 2017;29(1):26-34.

16. McGranahan N, Swanton C. Clonal Heterogeneity and Tumor Evolution: Past, Present, and the Future. Cell. 2017;168(4):613-628.

17. Testa U, Castelli G, Pelosi E. Lung Cancers: Molecular Characterization, Clonal Heterogeneity and Evolution, and Cancer Stem Cells. Cancers (Basel). 2018;10(8):248.

18. Lawson DA, Kessenbrock K, Davis RT, Pervolarakis N, Werb Z. Tumour heterogeneity and metastasis at single-cell resolution. Nat Cell Biol. 2018;20(12):1349-1360.

19. Schettini F, Prat A. Dissecting the biological heterogeneity of HER2-positive breast cancer. Breast. 2021;59:339-350. 
20. Wu H, Wang Q, Liu Q, Zhang Q, Huang Q, Yu Z. The Serum Tumor Markers in Combination for Clinical Diagnosis of Lung Cancer. Clin Lab. 2020;66(3).

21. Zaleski M, Kobilay M, Schroeder L, Debald M, Semaan A, Hettwer K, Uhlig S, Kuhn W, Hartmann G, Holdenrieder S. Improved sensitivity for detection of breast cancer by combination of miR-34a and tumor markers CA 15-3 or CEA. Oncotarget. 2018;9(32):22523-22536.

22. Kobayashi T. A blood tumor marker combination assay produces high sensitivity and specificity for cancer according to the natural history. Cancer Med. 2018;7(3):549-556.

\section{Tables}

Table 1. Age and sex data for each group

\begin{tabular}{|c|c|c|c|c|c|}
\hline \multirow[t]{2}{*}{ Groups } & \multirow[t]{2}{*}{ Sex } & \multirow[t]{2}{*}{$\mathrm{N}$} & \multicolumn{2}{|c|}{ Age (y) } & \multirow[t]{2}{*}{$\mathrm{P}$} \\
\hline & & & Mean & SD & \\
\hline \multirow[t]{2}{*}{ Poor-differentiated } & Men & 31 & 64.2 & 9.5 & \\
\hline & Women & 23 & 68.9 & 11.4 & \\
\hline \multirow[t]{2}{*}{ Well-differentiated } & Men & 33 & 66.1 & 9.5 & 0.220 \\
\hline & Women & 23 & 66.4 & 14.4 & \\
\hline \multirow[t]{2}{*}{ Benign lesions } & Men & 29 & 64.1 & 11.6 & \\
\hline & Women & 23 & 62.9 & 13.6 & \\
\hline \multirow[t]{2}{*}{ Healthy } & Men & 14 & 61.1 & 6.9 & \\
\hline & Women & 17 & 61.8 & 6.6 & \\
\hline
\end{tabular}

Table 2. Raw data on tumor marker levels in different groups (mean \pm SD) 


\begin{tabular}{|llllll|}
\hline \multirow{2}{*}{ Biomarkers } & \multicolumn{2}{l}{ Cancers (differentiated) } & Benign lesions & Healthy control & $P$ \\
\cline { 2 - 5 } & Poor & Well & & \\
\hline CEA & $50.2 \pm 142.5$ & $11.7 \pm 47.2$ & $2.5 \pm 5.3$ & $2.0 \pm 1.1$ & $<0.001$ \\
\hline AFP & $67.1 \pm 263.4$ & $17.7 \pm 81.1$ & $3.6 \pm 1.9$ & $3.9 \pm 2.0$ & 0.558 \\
\hline CA199 & $254.9 \pm 554.0$ & $49.1 \pm 264.1$ & $11.1 \pm 11.1$ & $11.1 \pm 7.2$ & $<0.001$ \\
\hline CA724 & $19.9 \pm 53.4$ & $9.6 \pm 39.7$ & $9.1 \pm 14.6$ & $3.9 \pm 2.0$ & $<0.001$ \\
\hline NSE & $18.2 \pm 35.3$ & $10.4 \pm 9.1$ & $7.6 \pm 4.8$ & $7.4 \pm 4.2$ & 0.014 \\
\hline FER & $300.1 \pm 329.8$ & $162.7 \pm 212.1$ & $131.8 \pm 204.3$ & $110.1 \pm 40.5$ & $<0.001$ \\
\hline CRP & $78.4 \pm 80.9$ & $20.9 \pm 28.0$ & $9.2 \pm 14.9$ & $3.1 \pm 2.3$ & $<0.001$ \\
\hline
\end{tabular}

Table 3. Positivity rate of the tumor markers in different groups (\%)

\begin{tabular}{|llllll|}
\hline Biomarkers & \multicolumn{2}{l}{ Cancers (differentiated) } & Benign lesions & Healthy control & $P$ \\
\cline { 2 - 4 } & Poor & Well & & & \\
\hline CEA & 51.9 & 25.0 & 5.8 & 0 & $<0.001$ \\
\hline AFP & 14.8 & 5.4 & 3.8 & 0 & 0.033 \\
\hline CA199 & 40.7 & 8.9 & 5.8 & 0 & $<0.001$ \\
\hline CA724 & 55.6 & 19.6 & 40.4 & 0 & $<0.001$ \\
\hline NSE & 18.5 & 5.4 & 0 & 0 & $<0.001$ \\
\hline FER & 42.6 & 16.1 & 5.8 & 3.2 & $<0.001$ \\
\hline CRP & 70.4 & 57.1 & 23.1 & 0 & $<0.001$ \\
\hline
\end{tabular}

Table 4. Positivity rate for the number of biomarkers present in different groups (\%) 


\begin{tabular}{|lllll|}
\hline \multirow{2}{*}{ Number of positive biomarkers } & \multicolumn{2}{l}{ Cancers (differentiated) } & Benign lesions & Healthy control \\
\cline { 2 - 4 } & Poor & Well & & \\
\hline 0 & 13.0 & 23.2 & 46.2 & 96.8 \\
\hline 1 & 23.2 & 33.9 & 38.5 & 3.2 \\
\hline 2 & 13.0 & 30.4 & 5.8 & 0 \\
\hline 3 & 20.4 & 8.9 & 3.8 & 0 \\
\hline 4 & 22.2 & 1.8 & 5.8 & 0 \\
\hline 5 & 14.8 & 1.8 & 0 & 0 \\
\hline 6 & 5.6 & 0 & 0 & 0 \\
\hline 7 & 0 & 0 & 0 & 0 \\
\hline
\end{tabular}

Table 5. Cancer molecular index in different groups

\begin{tabular}{|llllll|}
\hline Quartile & \multicolumn{2}{l}{ Cancers (differentiated) } & Benign lesions & Healthy control & $R(P)$ \\
\cline { 2 - 4 } & Poor & Well & & & \\
\hline 25 th & 3.5 & 1.1 & 0 & 0 & $0.647(<0.001)$ \\
\hline 50 th & 23.2 & 2.7 & 1.1 & 0 & \\
\hline 75 th & 117.6 & 7.3 & 1.6 & 0 & \\
\hline
\end{tabular}

Figures 


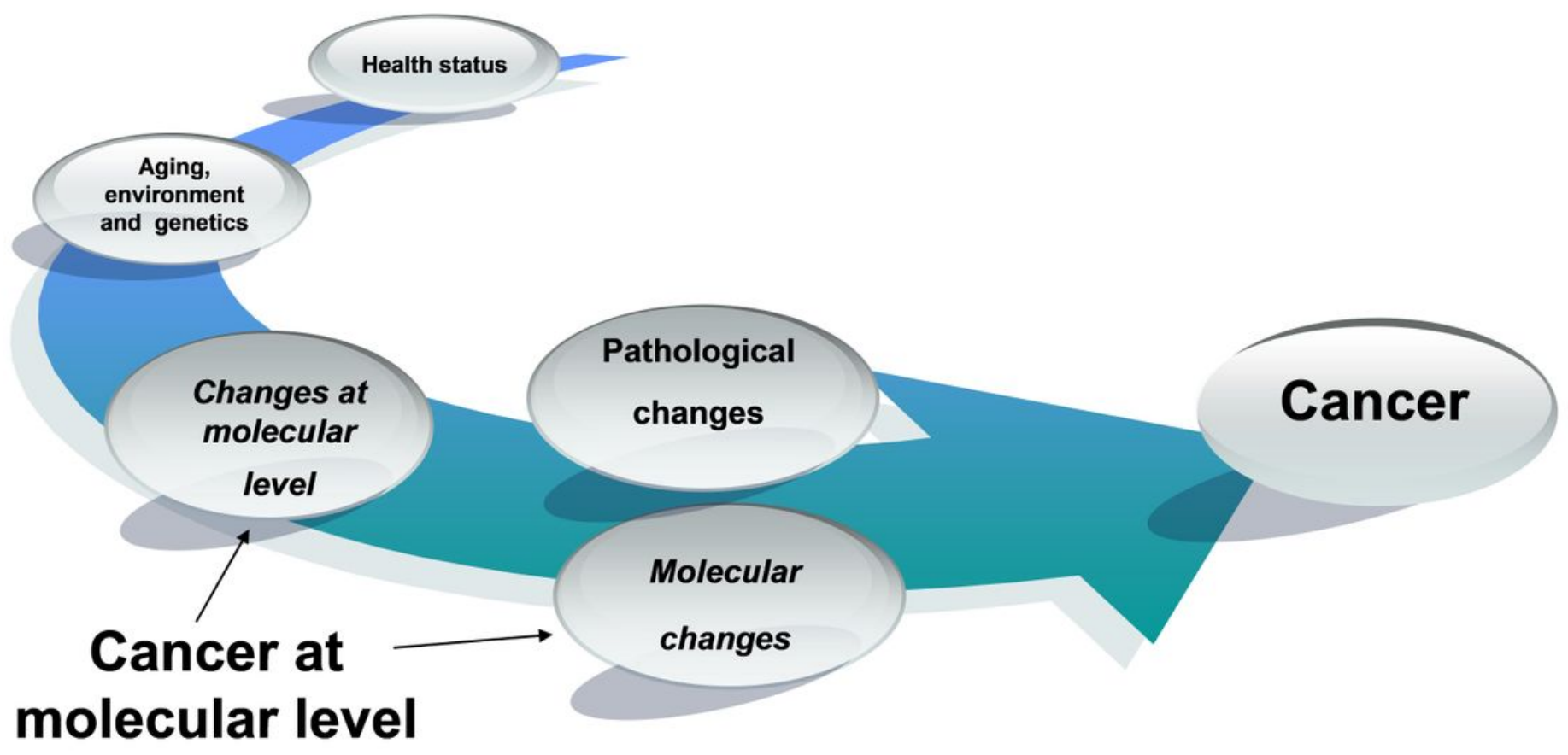

Figure 1

Morphological and molecular change associated with tumorigenesis

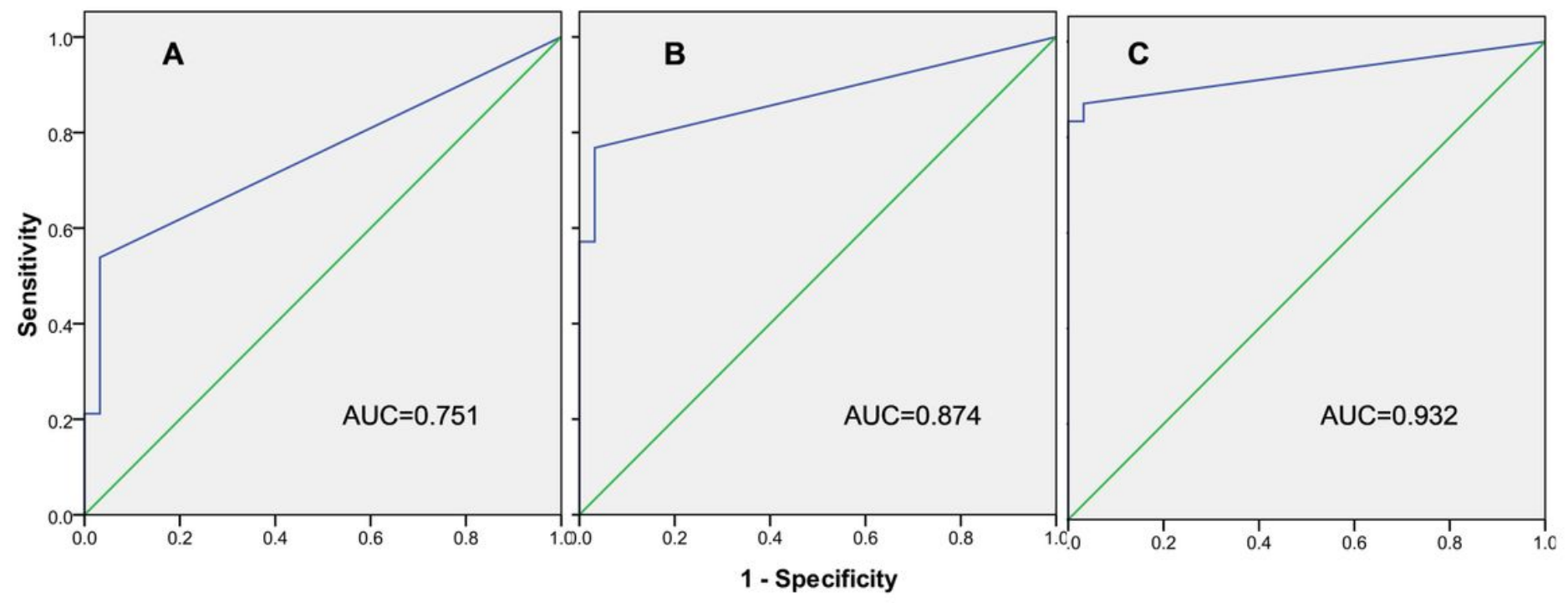

Figure 2

ROC curve for different groups for various cutoff levels of the cancer marker index (CMI) 


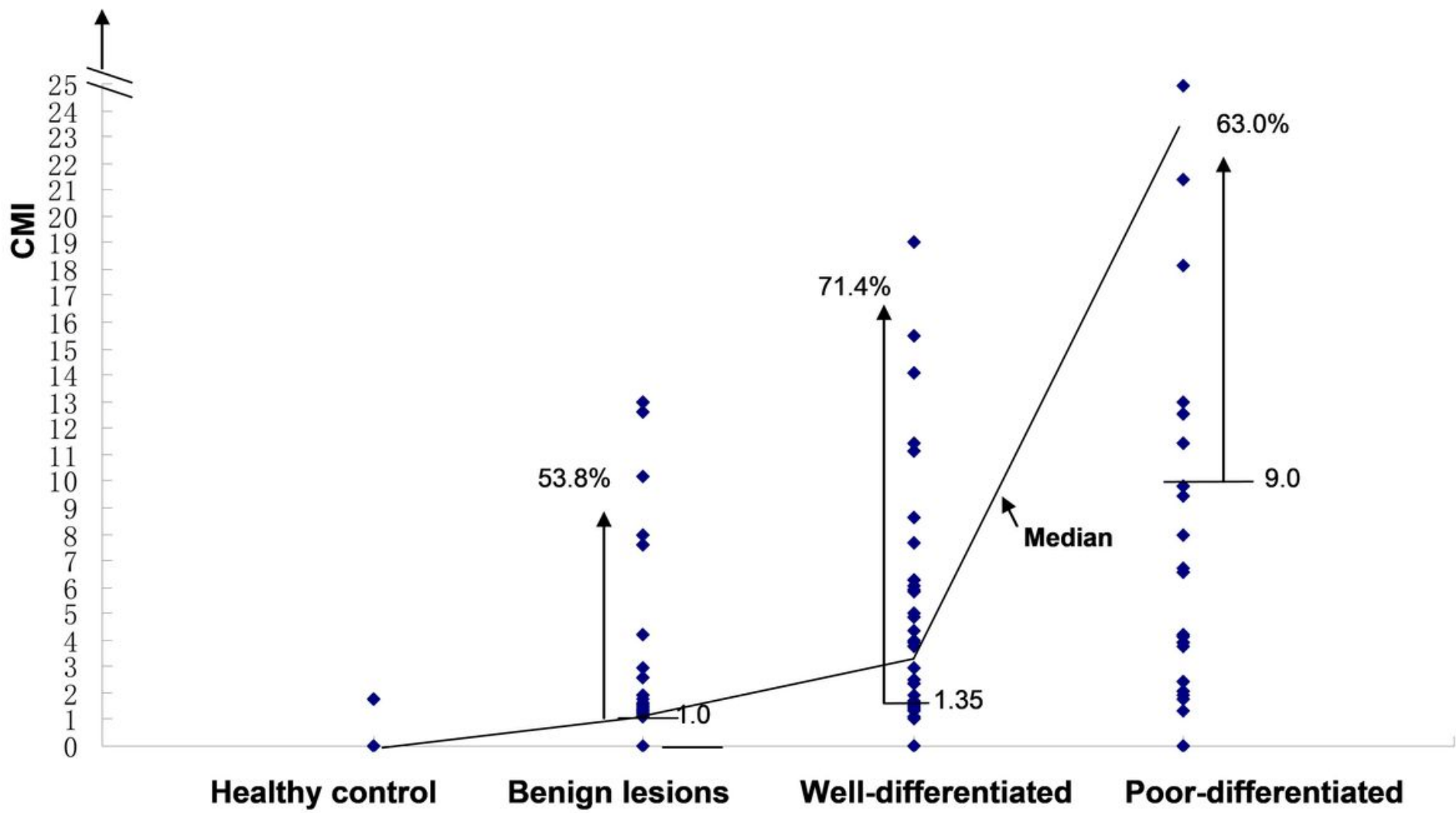

Figure 3

Cut-off levels of the cancer marker index (CMI)

The cut-off level of CMI was 1.00 for benign lesions, 1.35 for well-differentiated tumors, and 9.00 for poorly differentiated tumors. 